\title{
Electric field mediated droplet centering
}

\author{
Z.-M. Bei, ${ }^{1}$ T. B. Jones, ${ }^{1, a)}$ A. Tucker-Schwartz, ${ }^{2}$ and D. R. Harding ${ }^{3}$ \\ ${ }^{1}$ Department of Electrical and Computer Engineering, University of Rochester, \\ Rochester, New York 14627, USA \\ ${ }^{2}$ Department of Chemistry and Biochemistry, UCLA, Los Angeles, California 90095, USA \\ ${ }^{3}$ Laboratory for Laser Energetics and Department of Chemical Engineering, University of Rochester, \\ Rochester, New York 14623, USA
}

(Received 22 September 2008; accepted 14 October 2008; published online 3 November 2008)

\begin{abstract}
Double emulsion droplets subjected to a uniform ac electric field self-assemble into highly concentric structures via the dipole/dipole force if the outer droplet has a higher dielectric constant than the suspending liquid. The dielectric constant of the inner droplet has no influence. To minimize field-induced droplet distortion, the liquids must be density matched to $\sim 0.1 \%$. Centering of $\sim 3$ to $6 \mathrm{~mm}$ diameter droplets is achieved within $\sim 60$ s for field strengths of $\sim 10^{4} \mathrm{~V}_{\mathrm{rms}} / \mathrm{m}$ in liquids of viscosity $\sim 10 \mathrm{cP}$. Effective centering depends strongly on frequency if the outer shell is conductive. (C) 2008 American Institute of Physics. [DOI: 10.1063/1.3013577]
\end{abstract}

Many fields of science and technology employ doubleemulsion droplets, that is, droplets of one liquid contained within a droplet of another immiscible liquid. One application is in the formation of foam shells for laser fusion targets. These shells, typically $\sim 3 \mathrm{~mm}$ in diameter with $250 \mu \mathrm{m}$ wall thickness, , are formed from double emulsion droplets coming from a triple nozzle device. The droplets are stirred to center the inner droplet within the outer, then heated to initiate polymerization. ${ }^{2-4}$ This empirical process does produce acceptable foam shells but the yield is very low. Most of the shells fail to meet the $\sim 1 \%$ concentricity requirement. To produce $\sim 10^{4}$ targets per hour for laser fusion based power generation, higher yields are essential. A microfluidic assembly line is a possible solution if the droplet-centering problem can be addressed. ${ }^{5}$ Electrostatic self-assembly may fulfill this need.

Consider a uniform ac electric field imposed on a double-emulsion droplet in a third suspending liquid (see Fig. 1). Elementary energy considerations dictate that, when located at the geometric center of the outer shell, the inner droplet will be either stable or unstable. The necessary condition for stability is $\kappa_{2}>\kappa_{1}$, where $\kappa_{1}$ and $\kappa_{2}$ are, respectively, the dielectric constants of the suspending medium and liquid comprising the shell. One may validate this condition using the method of images. There is no closed-form image theory for dielectric spheres, ${ }^{6}$ but one may approximate an electrostatic solution with the lead image term. Imagine replacing the inner droplet with a test dipole $p_{\mathrm{o}}$ aligned with the $z$-axis and located within the outer sphere. Figure 2, which displays the signs of the lead image dipole terms for axial $(\delta z)$ and equatorial $(\delta x)$ displacements, teaches that the force is always restoring if $\kappa_{2}>\kappa_{1}$; in fact, a stable equilibrium is assured for displacements at any angle. The sign of the effective dipole induced in the inner droplet actually depends on $\kappa_{3}-\kappa_{2}$, but the sign of the product of this moment and its image - and therefore the force-does not. Thus, $\kappa_{3}$ does not influence the stability condition.

We numerically computed the electrostatic force on the inner droplet for selected values of $\kappa_{1}, \kappa_{2}$, and $\kappa_{3}$ using the

\footnotetext{
a) Author to whom correspondence should be addressed. Electronic mail: jones@ece.rochester.edu.
}

Maxwell stress tensor postprocessing feature of COMSOL MULTIPHYSICS ${ }^{\circledR}$. Figure 3 plots force versus axial displacement $\delta z$. Only curves for $\kappa_{2}>\kappa_{1}$ exhibit negative slope, corresponding to stability. Calculations for $\delta x$ displacement give the same result, except that the force magnitude is smaller by a factor of 0.75 . Predictions based on the first term of a multipolar expansion, ${ }^{7}$ also plotted in Fig. 3, prove accurate for small displacements.

For effective centering, the E-field must penetrate the shell to induce a strong dipole moment $p_{\text {eff }}$ in the inner droplet. However for foam shell formation, the liquid in the shells must contain monomers, which increase the electrical conductivity. The resultant electrostatic shielding can be overcome only by raising the frequency. The complex effective dipole moment of the inner droplet is ${ }^{8}$

$$
\begin{aligned}
\underline{p}_{\text {eff }}= & 12 \pi \varepsilon_{2} R_{\text {inner }}^{3} \\
& \times \frac{\underline{\varepsilon}_{1}\left(\underline{\varepsilon}_{2}-\underline{\varepsilon}_{3}\right)}{2\left(R_{\text {inner }} / R_{\text {outer }}\right)^{3}\left(\underline{\varepsilon}_{1}-\underline{\varepsilon}_{2}\right)\left(\underline{\varepsilon}_{2}-\underline{\varepsilon}_{3}\right)+\left(2 \underline{\varepsilon}_{1}+\underline{\varepsilon}_{2}\right)\left(2 \underline{\varepsilon}_{2}+\underline{\varepsilon}_{3}\right)} E_{0},
\end{aligned}
$$

where $\underline{\varepsilon}=\varepsilon+\sigma / \mathrm{j} \omega, \varepsilon$ is the permittivity, $\sigma$ is the electrical conductivity, $\omega$ is the radian frequency, $\mathrm{j}=\sqrt{-1}$, and $E_{\mathrm{o}}$ is the external ac field. Figure 4 plots $\left|p_{\text {eff }}\right|$ versus frequency for a typical experimental droplet. Because the force is proportional to $p_{\text {eff }}^{2}$, centering depends strongly on frequency. For a

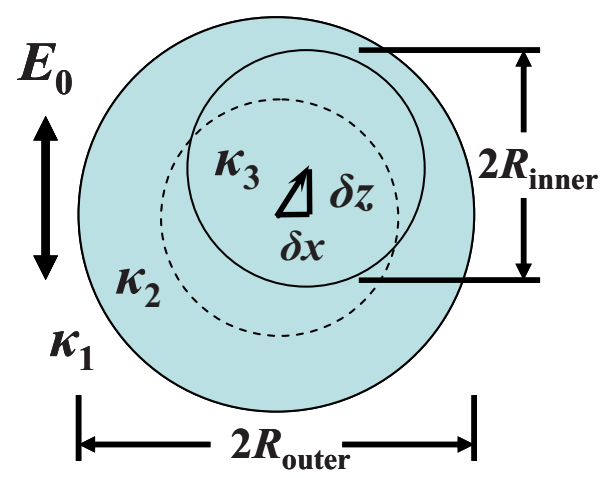

FIG. 1. (Color online) Double emulsion droplet immersed in a uniform ac electric field $E_{\mathrm{o}}$ with definitions provided for dielectric constants, radii, and displacements of inner droplet, $\delta z$ and $\delta x$. 


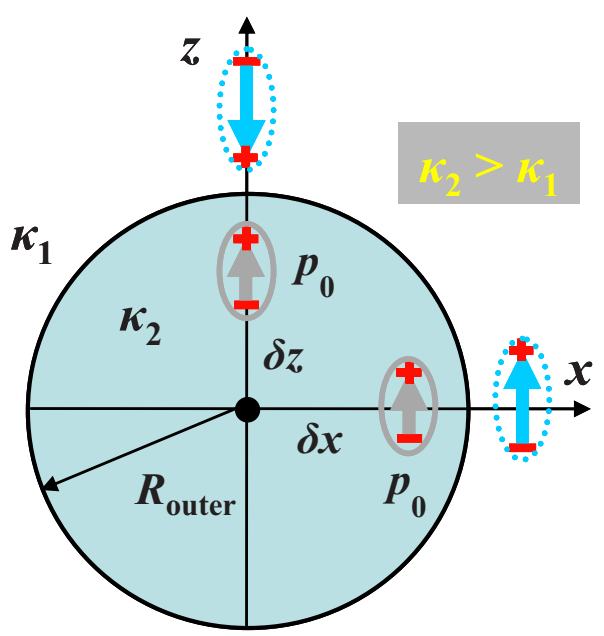

FIG. 2. (Color online) Polarities of dipole images induced by test dipole $p_{\mathrm{o}}$ for axial $(\delta z)$ and equatorial $(\delta x)$ displacement for the stable case, $\kappa_{2}>\kappa_{1}$.

laser target, the shell thickness is very thin, i.e., $d=R_{\text {outer }}$ $-R_{\text {inner }} \ll R_{\text {outer }}$ and also $\kappa_{2} \gg \kappa_{1}, \kappa_{3}$, so the critical frequency is $\omega_{\text {crit }} \approx 2 \sigma_{2} d / \varepsilon_{3} R_{\text {outer }}$. To circumvent shielding, $\omega \geq \omega_{\text {crit }}$.

To study centering dynamics, we developed a reducedorder model. On the time scale of interest for centering, inertia is negligible. Thus, the equation of motion for the inner droplet balances the electrostatic force against viscous drag.

$$
\alpha \delta z-\left(\beta+\zeta \delta z^{\lambda}\right) \frac{d \delta z}{d t}=0,
$$

where

$$
\delta_{z} \ll d .
$$

Coefficient $\alpha$ represents the centering force while $\beta, \zeta$, and $\lambda$ are obtained from a power law fit of viscous drag at low Reynolds and capillary numbers. ${ }^{9,10}$ An implicit solution for $\delta z(t)$ is readily obtained by integration of Eq. (2).

We conducted experiments to demonstrate electric field induced centering using a rectangular liquid-filled chamber fitted with parallel horizontal electrodes at the top and bottom. Nearly buoyant droplets are supported midway between the electrodes on a thin indium-tin-oxide (ITO) coated glass plate treated with Teflon-AFTM parallel to the electrodes. The suspending liquid and the inner droplet were mixtures

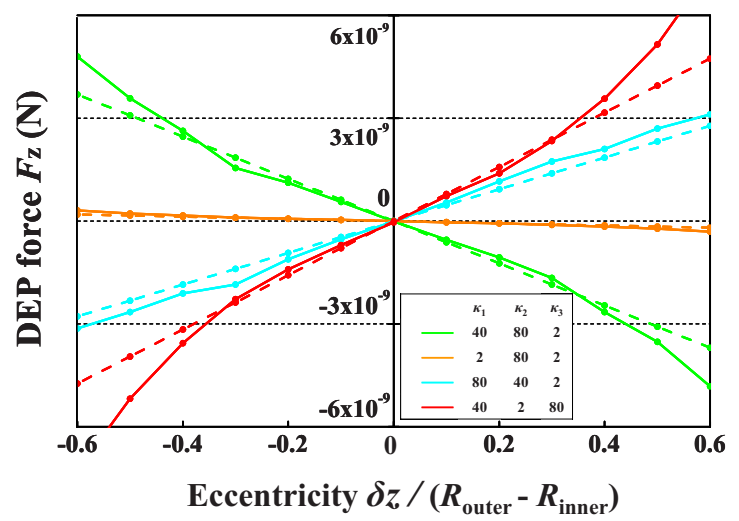

FIG. 3. (Color online) Computed dielectric force vs axial displacement expressed in terms of eccentricity $\delta z /\left(R_{\text {outer }}-R_{\text {inner }}\right)$ for selected values of $\kappa_{1}$, $\kappa_{2}$, and $\kappa_{3}$. $R_{\text {outer }}=1.5 \mathrm{~mm}, R_{\text {inner }}=1.0 \mathrm{~mm} ; E_{0} \sim 10^{4} \mathrm{~V}_{\text {rms }} / \mathrm{m}$. Solid lines: numerically computed electrostatic force. Dashed lines: force computed from analytical prediction based on multipolar expansion (Ref. 7).

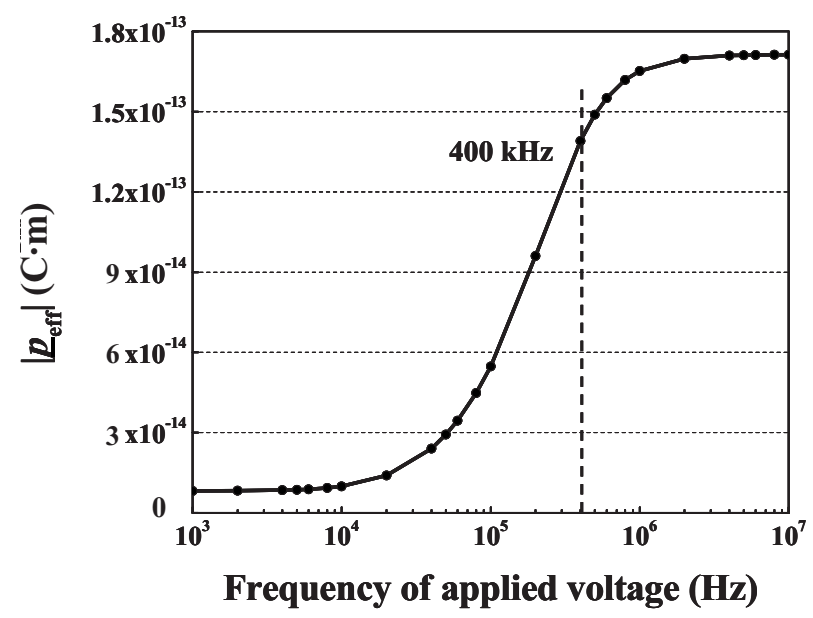

FIG. 4. Frequency dependence of the induced effective dipole moment $\left|p_{\text {eff }}\right|$ of the inner droplet with an estimate for the critical frequency $f_{\text {crit. }}$. System parameters: $\kappa_{1}=\kappa_{3}=2.5, \quad \kappa_{2}=37.8 ; \quad R_{\text {outer }}=2.9 \mathrm{~mm}, R_{\text {inner }}=2.2 \mathrm{~mm} ; \sigma_{1}$ $\sim \sigma_{3} \leq 2 \cdot 10^{-6} \mathrm{~S} / \mathrm{m}, \sigma_{2}=9.43 \cdot 10^{-4} \mathrm{~S} / \mathrm{m} ; E_{0}=3 \cdot 10^{4} \mathrm{~V}_{\mathrm{rms}} / \mathrm{m}$.

of $10 \mathrm{cS}\left(0.934 \mathrm{~g} / \mathrm{cm}^{3}\right)$ and $50 \mathrm{cS}\left(0.960 \mathrm{~g} / \mathrm{cm}^{3}\right)$ silicone oils (DC-200: Dow Corning). We selected N,Ndimethylacetamide or DMA (Alfa Aesar), an organic solvent immiscible in silicone oil, as the liquid for the outer shell because its density, $0.941 \mathrm{~g} / \mathrm{cm}^{3}$, is bracketed by the two silicone oils. For each experiment, a DMA droplet is dispensed on the holder; then, a silicone oil droplet is injected into it with a micromanipulator-mounted syringe. To prevent droplets from coalescence, we dope the DMA with surfactant (Span 80, Sigma-Aldrich).

We demonstrated the effect of shielding on centering by varying frequency at fixing $E_{\mathrm{o}}\left(\approx 1.3 \times 10^{4} \mathrm{~V}_{\mathrm{rms}} / \mathrm{m}\right)$ with a $670 \mu \mathrm{m}$ thick DMA shell of outer radius $2.9 \mathrm{~mm}$ and conductivity $\sigma_{2} \sim 10^{-3} \mathrm{~S} / \mathrm{m}$. At $20 \mathrm{kHz}$, the inner silicone oil droplet remained near the bottom of the outer droplet, but when the frequency was raised to $200 \mathrm{kHz}$, a value approaching the calculated $f_{\text {crit }}=\omega_{\text {crit }} / 2 \pi=400 \mathrm{kHz}$, it immediately rose to the center where it resided stably. Upon decreasing the frequency to $20 \mathrm{kHz}$, the droplet sank back to the bottom.

The electric field used to achieve centering causes ellipsoidal distortions ${ }^{11,12}$ of the order of $\leq 1 \%$. Interestingly enough, the larger distortions observed at high voltages might be exploited to accelerate centering. In one experiment with a $640 \mu \mathrm{m}$ thick DMA shell with an outer radius of 2.9 $\mathrm{mm}, 500 \mathrm{~V}_{\mathrm{rms}}$ at $100 \mathrm{kHz}$ was applied for $10 \mathrm{~s}$, then reduced to $280 \mathrm{~V}_{\mathrm{rms}}$. During the initial $10 \mathrm{~s}$, the inner droplet rapidly moved to the center, presumably due to strong viscous shear. When the voltage was reduced, the shell returned to spherical shape, retaining good concentricity. The short-term high voltage application reduced the centering time from $\sim 80$ to $<45$ s.

Using a focused beam from a light-emitting diode source to back light the droplets, we obtained videos adequate for analysis of the centering dynamics. For each video frame, a MATLAB ${ }^{\circledR}$ program uses edge detection to fit ellipsoids, one each to the outer shell profile and to the visible bright ring (caustic) of the inner droplet, and then computes the locations of the centers. The program determines the ratio of the semimajor to semiminor axes, $a / b$, and superimposes the fitted ellipses upon the original video frames. 


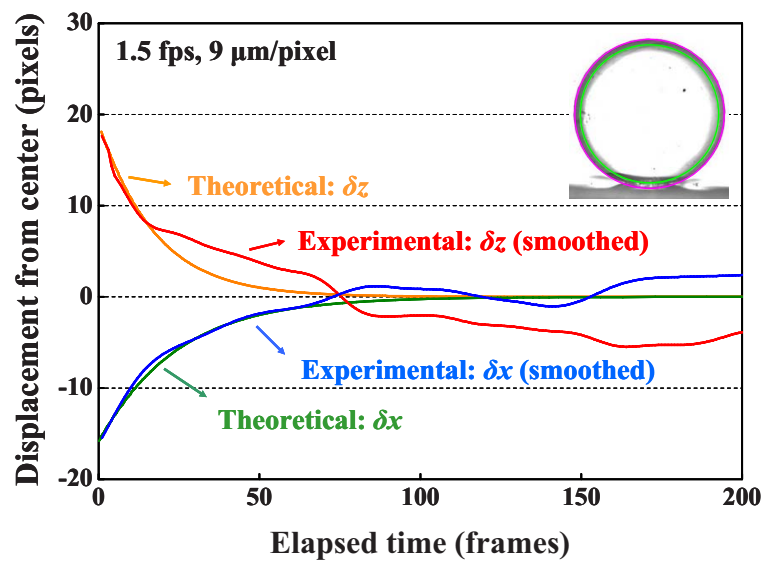

FIG. 5. (Color online) Axial $(\delta z)$ and equatorial ( $\delta x)$ centering dynamics of the inner droplet. Red and blue curves are FFT smoothed data from MAT$\mathrm{LAB} ®$ tracking program. Orange and green curves are predictions from Eq. (2). Experimental parameters: $\kappa_{1}=\kappa_{3}=2.5, \kappa_{2}=37.8, \mu_{1}=10.5 \mathrm{cP}, \mu_{2}$ $=1.96 \mathrm{cP}, \mu_{3}=13.2 \mathrm{cP}$, DMA volume $=55 \mu \mathrm{l}$, volume of inner silicone oil droplet $=100 \mu \mathrm{l}, R_{\text {inner }}=2.9 \mathrm{~mm}, R_{\text {outer }}=3.3 \mathrm{~mm}, E_{0}=3 \times 10^{4} \mathrm{~V}_{\text {rms }} / \mathrm{m}$, optical resolution $=9 \mu \mathrm{m} /$ pixel, and frame rate $=1.5 \mathrm{fps}$.

Figure 5 plots fast Fourier transform (FFT)-smoothed experimental data for axial and equatorial centering of a double emulsion droplet. Centering occurs in $\sim 60 \mathrm{~s}$ for $E_{\mathrm{o}}$ $=3 \times 10^{4} \quad \mathrm{~V}_{\mathrm{rms}} / \mathrm{m}$. Equatorial motion of the inner droplet is fairly consistent with the predictions of the dynamic model of Eq. (2). On the other hand, along the vertical axis, the inner droplet settles $\sim 45 \mu \mathrm{m}(\sim 5$ pixels $)$ below the center, probably due to the effect of the conductive ITO holder upon the electric field. This effect diminishes as the shell thickness is reduced. We are now testing a density gradient suspension scheme to eliminate the holder altogether.

In conclusion, effective droplet centering using a uniform applied ac electric field of magnitude $\sim 10^{4} \mathrm{~V}_{\text {rms }} / \mathrm{m}$ occurs in $\sim 60 \mathrm{~s}$. Some droplet distortions are evident and the ITO glass holder displaces the vertical equilibrium downward. For shell conductivities $>10^{-3} \mathrm{~S} / \mathrm{m}$, electrostatic shielding requires frequencies $\geq 1 \mathrm{MHz}$. At these higher conductivities, Ohmic heating and thermal convection may start to interfere with electrostatic centering.

M. Washizu (University of Tokyo) provided us with a multipolar representation of the electrostatics of doubleemulsion droplets. P. Osborne, M. Wittman, and W. Bittle (University of Rochester) built and assembled much of the experimental apparatus. D. Jacobs-Perkins (Rochester) provided the MATLAB ${ }^{\circledR}$ image analysis program. This work was supported by the U.S. Department of Energy Office of Inertial Confinement Fusion, the Laboratory for Laser Energetics at the University of Rochester, the New York State Energy Research and Development Authority, and General Atomics.

${ }^{1}$ K. R. Schulz, Proceedings of the IEEE NPSS Symposium on Fusion Engineering, 1995 (unpublished), Vol. 1, p. 116.

${ }^{2}$ S. M. Lambert, G. E. Overturf, G. Wilemsk, S. A. Letts, D. SchroenCarey, and R. C. Cook, J. Appl. Polym. Sci. 65, 2111 (1997).

${ }^{3}$ R. R. Paguio, A. Nikroo, J. F. Hund, C. A. Frederick, J. Jaquez, M. Takagi, and M. Thi, MRS Symposia Proceedings No. 901E (Materials Research Society, Pittsburgh, 2006), p. 78.

${ }^{4}$ R. R. Paguio, A. Nikroo, M. Takagi, and O. Acenas, J. Appl. Polym. Sci. 101, 2523 (2006).

${ }^{5}$ D. R. Harding, T. B. Jones, Z.-M. Bei, D. Edgell, and S. H. Chen, Cryogenic-DT-Foam Targets: The New Frontier, Proceedings of the 18th Target Fabrication Mtg, Lake Tahoe, CA, 2008 (unpublished).

${ }^{6} \mathrm{E}$. Weber, Electromagnetic Theory, Static Fields and Their Mapping (Dover, New York, 1965), pp. 228-233.

${ }^{7}$ M. Washizu, personal communication (17 October 2007).

${ }^{8}$ T. B. Jones, Electromechanics of Particles (Cambridge University Press, New York, 1995), pp. 227-230.

${ }^{9}$ S. S. Sadhal and H. N. Oguz, J. Fluid Mech. 160, 511 (1985).

${ }^{10}$ M. Stimson and G. B. Jeffery, Proc. R. Soc. London, Ser. A 111, 110 (1926)

${ }^{11}$ C. G. Garton and Z. Krasucki, Proc. R. Soc. London, Ser. A 280, 211 (1964).

${ }^{12}$ C. T. O'Konski and H. C. Thacher, J. Phys. Chem. 57, 955 (1953). 